\title{
Menstrual cycle phase effects free testosterone responses to prolonged aerobic exercise (Short communication)
}

\author{
AR Lane, CB O'Leary, AC Hackney \\ Endocrine Section - Applied Physiology Laboratory, Department of Exercise \& Sport Science, \\ University of North Carolina, Chapel Hill, NC, USA \\ Department of Nutrition, Gillings School of Global Public Health, University of North Carolina, \\ Chapel Hill, NC, USA
}

Received: May 7, 2015

Accepted after revision: June 9, 2015

\begin{abstract}
Research has shown that total testosterone (tT) levels in women increase acutely during a prolonged bout of aerobic exercise. Few studies, however, have considered the impact of the menstrual cycle phase on this response or have looked at the biologically active free testosterone (fT) form responses. Therefore, this study examined the fT concentration response independently and as a percentage ( $\mathrm{fT} \%$ ) of t to prolonged aerobic exercise during phases of the menstrual cycle with low estrogen-progesterone (L-EP; i.e., follicular phase) and high estrogen-progesterone (H-EP; i.e., luteal phase). Ten healthy, recreationally trained, eumennorrheic women $(\mathrm{X} \pm \mathrm{SD}$ : age $=20 \pm 2 \mathrm{y}$, mass $=58.7 \pm 8.3 \mathrm{~kg}$, body fat $=22.3 \pm 4.9 \%, \mathrm{VO}_{2 \max }=50.7 \pm 9.0 \mathrm{ml} / \mathrm{kg} / \mathrm{min}$ ) participated in a laboratory based study and completed a 60 -minute treadmill run during the L-EP and $\mathrm{H}$-EP menstrual phases at $\sim 70 \% \mathrm{of} \mathrm{VO}_{2 \max }$. Blood was drawn prior to (PRE), immediately after (POST) and following 30 minutes of recovery (30POST) with each 60-minute run. During H-EP, there was a significant increase in fT concentrations from PRE to POST $(p<0.01)$ while in L-EP fT levels were unchanged; which resulted in $\mathrm{fT}$ being significantly higher at H-EP POST versus L-EP POST $(p<0.03)$. Area-under-the-curve (AUC) responses were calculated, for fT the total AUC was greater in H-EP than L-EP $(p<0.04)$. There was no significant interaction of $\mathrm{fT} \%$ between phases and exercise sampling time. There was, however, a main effect for exercise where fT\% POST was a greater proportion of tT than at PRE $(p<0.01)$. In summary, hormonal changes associated with the menstrual cycle impact fT response to a prolonged aerobic exercise bout; specifically, there being higher levels under H-EP conditions. This suggests more biologically active $\mathrm{T}$ is available during exercise in this phase. This response may be a function of the higher core temperatures found with H-EP causing greater sex hormone binding protein release of $\mathrm{T}$, or could be a function of greater degrees of glandular production. Further work is warranted to elucidate the mechanism of this occurrence. It is recommended that researchers examining $\mathrm{T}$ responses to exercise in women look at both tT and fT forms in order to have an accurate endocrine assessment in women.
\end{abstract}

Keywords: endocrine, menstrual cycle, women, training, androgens, hormone

Research has shown that total testosterone (tT) levels in women increase acutely during a prolonged bout of aerobic exercise (8), although few studies have addressed this response thoroughly $(1,7)$. Consideration of the influence of the menstrual cycle is important due to the fact that testosterone levels and production can change dramatically over the cycle (5). Interestingly, even fewer exercise studies have examined the more important biologically active form of testosterone (i.e., free testosterone [fT]) response with regard to menstrual

Corresponding author: Anthony C. Hackney

CB \#8700, Fetzer Hall, University of North Carolina

Chapel Hill, NC 27599, USA

Phone: +1-919-962-0334; Fax: +1-919-962-0489; E-mail: ach@email.unc.edu 
cycle phase $(5,8)$. Regrettably, some investigators have made the assumption that fT responses mirror those of $\mathrm{tT}$, hence measuring only one form of the hormones is necessary (N.B., usually tT is typically measured due to assay cost considerations). Given the endocrinological differences within the body of a woman between the low-estrogen progesterone follicular phase and high-estrogen progesterone luteal phase of the menstrual cycle, it is possible the fT does not mimic as closely the tT responses as expected when coupled with the physical stress of exercise. This point seems especially valid in light of the role that estrogens and progesterone can have in the regulatory aspects of testosterone and sex-hormone binding globulin production (5). In light of the short-comings in the available research studies in this area, the present study was conducted to examine and compare the fT responses to prolonged aerobic exercise during the low estrogen-progesterone (L-EP) follicular and higher estrogen-progesterone (H-EP) luteal phases of the menstrual cycle.

\section{Materials and Methods}

\section{Subjects}

Ten healthy, recreationally trained women $(20.0 \pm 2.2 \mathrm{yr}: \mathrm{X} \pm \mathrm{SD})$ volunteered to participate in this laboratory based original study. All were eumenorrheic without the use of hormonal contraceptives or any hormonal therapy within the 6 months preceding participation, as well as having a normal menstrual cycle of 24-35 days with a mean cycle length of $30 \pm 3$ days. Physical characteristics are as follows: height $=164.1 \pm 5.9 \mathrm{~cm}$, body mass $=58.7 \pm 8.3 \mathrm{~kg}$, body fat $=22.3 \pm 4.9 \%$ and $\mathrm{BMI}=21.8 \pm 3.1 \mathrm{~kg} / \mathrm{m}^{2}$.

\section{Procedures}

Each subject gave written informed consent prior to participation. This study was conducted according to the Declaration of Helsinki and was approved by the university ethics board.

Subjects reported to our laboratory on three different occasions. The initial session included obtaining written consent, familiarizing subjects with the testing protocol, recording anthropometrics, and performing a maximal cardiopulmonary aerobic exercise test $\left(\mathrm{VO}_{2 \max }\right)$. Anthropometrics included measuring height, body mass, and estimating body fat percent using skinfolds as previously reported (8) (Lange skinfold calipers; Cambridge Scientific Industries, Inc., Cambridge, MA).

The two subsequent exercise sessions each involving a 60-minute submaximal treadmill runs were performed. These were conducted on different days, one during the mid-follicular and the other during the mid-luteal phase of the menstrual cycle. Subjects informed the investigator of the first day of menses and the forward counting method was used to determine phase and scheduling. Mid-follicular (L-EP) was occurred $7 \pm 2$ days, and mid-luteal (H-EP) was $20 \pm 2$ days from the onset of menses. Urinary ovulation tests along with measured basal blood estrogen levels were utilized to confirm the appropriate menstrual cycle phase. Prior to arrival for the 60 minute exercise sessions, subjects were informed to avoid strenuous physical activity, drink sufficient water $(\sim 1.0 \mathrm{~L})$, refrain from caffeine and alcohol use and maintain a log of their diet to insure it was comprised of at least $60 \%$ carbohydrates for 24 hours prior to the session. Subjects were asked to arrive 2 hours post-prandial. Upon arrival to the laboratory, subjects were fitted with a heart rate monitor and asked to rest in a supine position for ten minutes to establish a resting heart rate (HR). 
As noted, each subject performed a $\mathrm{VO}_{2 \max }$ test, which used a Bruce treadmill protocol to volitional fatigue. Expired gases were measured continuously during the test with the Parvo Medics True Max ${ }^{\circledR} 2400$ Metabolic System (Parvo Medics, Salt Lake City, UT). Subject HR was monitored at the end of each minute of exercise with a Polar HR monitor (Polar Electro, Inc, Lake Success, NY) and rate of perceived exertion (RPE) was recorded in the final minute of each exercise stage. Three of the four following criteria were used to identify a maximal exercise effort: 1 . respiratory exchange rate $(\mathrm{RER}) \geq 1.1,2$. maximal HR $\pm 5 \%$ of age-predicted $\mathrm{HR}_{\max }, 3$. RPE $\geq 18$, and 4 . a decrease or plateau of the $\mathrm{VO}_{2}$ with an increase in workload (9).

Two 60-minute exercise sessions at $70 \% \mathrm{VO}_{2 \max }$, as determined from the ACSM metabolic calculations, were completed by all subjects (9). One during the mid-follicular (LEP) and one during the mid-luteal (H-EP) phase of the menstrual cycle. Upon arrival at the laboratory for these sessions, subjects were fitted with a HR monitor and began 10 minutes of supine rest. Following the rest, the subjects warmed up by walking easily on the treadmill, followed by stretching for 5 minutes. After the warm-up, subjects ran on the treadmill for 60 minutes at speed based upon their predetermined intensity and at $0 \%$ grade. To confirm the appropriate workload was being achieved, expired gases were collected and analyzed in four minute intervals at 6-10, 26-30, and 56-60 minutes of the runs. Heart rate and RPE were recorded at 10, 30, and 60 minutes of exercise. The respiratory exchange ratio (RER) was also recorded during each of the three-four-minute respiratory measurement periods. Subjects were allowed to drink water ad libitum throughout the 60-minute runs. At the completion of the exercise, subjects walked for 3-5 minutes to cool down followed by 30 minutes of supine rest. The exact same protocol was followed by each subject for both 60 minute exercise sessions, including the time of day for the exercise. All exercise and respiratory gas equipment were identical for the $\mathrm{VO}_{2 \max }$ testing sessions.

Blood specimen samples were collected at three separate time points during both 60 minute exercise sessions, prior to the exercise (PRE; following rest), immediately following exercise ending (POST), and 30 minutes into recovery (30POST). A 3-mL sample was obtained through a venous catheter placed in an antecubital vein. The blood was collected into $\mathrm{K}_{2}$-EDTA blood collection tubes and placed immediately on ice. Once the 30POST sample was collected, plasma samples were prepared, aliquotted into 2-mL cryovials, and stored in a $-80{ }^{\circ} \mathrm{C}$ freezer until later analysis. Conventional enzyme-linked immunosorbent assay (ELISA) kits were used to measure concentrations of estradiol (Abnova Inc., Walnut, $\mathrm{CA}$ ) as well as fT and tT (Abnova Inc., Walnut, CA). Intra-assay coefficients for estradiol, tT and $\mathrm{fT}$ were $8.1,10.8$, and $11.2 \%$, respectively.

\section{Statistical analysis}

SPSS software (version 19.0, Chicago, IL) was utilized to conduct all statistical analysis. The significance level was set $a$ priori at $\alpha<0.05$ and all values are reported as mean $\pm \mathrm{SD}$. Analyses were conducted using a 2 (L-EP, H-EP; phase) $\times 3$ (PRE, POST, 30POST; sampling time) repeated measures ANOVA to compare the impact of menstrual phase on $\mathrm{fT}$ and $\mathrm{tT}$ concentrations as well as the relative $\mathrm{fT}$ (fT/tT as a percentage [\%]) at each time point. Bonferroni post hoc-analysis was completed to identify specific statistically significant differences within ANOVAs. Additionally, tT and fT area-under-the-curve (AUC) responses were calculated for PRE to POST, and POST to 30POST sampling times as well as total AUC responses using all sampling times (TOTAL AUC). The AUC data were statistically analyzed using dependent $t$-tests. 


\section{Results}

For the maximal cardiopulmonary test the subject's $\mathrm{VO}_{2 \max }=50.7 \pm 9.0 \mathrm{ml} / \mathrm{kg} / \mathrm{min}$. Furthermore, all subjects reached the necessary three out of four criteria (i.e., HR, RER, RPE, and $\mathrm{VO}_{2}$ ) indicating a valid maximal $\mathrm{VO}_{2 \max }$ test occurred (9).

For the 60 minute exercise sessions the exercise intensities were; L-EP $=69.7 \pm 7.3 \%$ of $\mathrm{VO}_{2 \max }, \mathrm{H}-\mathrm{EP}=67.6 \pm 7.9 \%$ of $\mathrm{VO}_{2 \max }$, respectively; the exercise intensities for H-EP were not different from L-EP $(p>0.05)$. Physiological responses to the 60-minute exercise sessions for $\mathrm{HR}, \mathrm{VO}_{2}, \mathrm{RPE}$ and RER were comparable and not significantly different between sessions for any measure $([p>0.05]$ these data have been reported in detail elsewhere (8)).

Resting (PRE) estrogen levels were assessed before each of the 60-min submaximal sessions to confirm menstrual phase status; $27.0 \pm 6.1 \mathrm{pg} / \mathrm{mL}$ during L-EP and $67.6 \pm 21.7 \mathrm{pg} /$ $\mathrm{mL}$ during H-EP $(p<0.05)$. The hormonal responses before and after the 60 -minute exercise sessions for $\mathrm{tT}$, fT, and relative $\mathrm{fT} \%$ can be found in Table I.

Table I. Response (mean $\pm \mathrm{SD}$ ) of total, free, and relative free testosterone to 60 -minute treadmill running sessions in the H-EP and L-EP phases of the menstrual cycle.

\begin{tabular}{|c|c|c|c|c|c|c|}
\hline \multicolumn{7}{|c|}{ Hormonal concentrations } \\
\hline \multirow{2}{*}{$\begin{array}{c}\text { Measurement } \\
\text { time }\end{array}$} & \multicolumn{3}{|c|}{ Mid-luteal (H-EP) } & \multicolumn{3}{|c|}{ Mid-follicular (L-EP) } \\
\hline & $\begin{array}{c}\mathrm{tT} \\
(\mathrm{pg} / \mathrm{ml})\end{array}$ & $\begin{array}{c}\text { fT } \\
(\mathrm{pg} / \mathrm{ml})\end{array}$ & $\begin{array}{c}\% f^{a} \\
(\%)\end{array}$ & $\begin{array}{c}\text { tT } \\
(\mathrm{pg} / \mathrm{ml})\end{array}$ & $\begin{array}{c}\mathrm{fT} \\
(\mathrm{pg} / \mathrm{ml})\end{array}$ & $\begin{array}{c}\% f^{a} \\
(\%)\end{array}$ \\
\hline PRE & $\begin{array}{c}368 \\
\pm 211\end{array}$ & $\begin{array}{c}1.129 \\
\pm 0.907\end{array}$ & $\begin{array}{c}0.282 \\
\pm 0.171\end{array}$ & $\begin{array}{c}409 \\
\pm 199\end{array}$ & $\begin{array}{c}1.259 \\
\pm 0.888\end{array}$ & $\begin{array}{l}0.270 \\
\pm 0.098\end{array}$ \\
\hline POST & $\begin{array}{l}702 * \\
\pm 519\end{array}$ & $\begin{array}{l}3.872 * \mathrm{~d} \\
\pm 3.588\end{array}$ & $\begin{array}{c}0.465 \\
\pm 0.168\end{array}$ & $\begin{array}{l}537^{*} \\
\pm 199\end{array}$ & $\begin{array}{c}1.796^{\mathrm{d}} \\
\pm 0.907\end{array}$ & $\begin{array}{l}0.312 \\
\pm 0.068\end{array}$ \\
\hline 30POST & $\begin{array}{c}488 \\
\pm 314\end{array}$ & $\begin{array}{c}2.365 \\
\pm 1.826\end{array}$ & $\begin{array}{c}0.445 \\
\pm 0.201\end{array}$ & $\begin{array}{c}505 \\
\pm 300\end{array}$ & $\begin{array}{c}1.587 \\
\pm 1.310\end{array}$ & $\begin{array}{l}0.270 \\
\pm 0.087\end{array}$ \\
\hline
\end{tabular}

$\mathrm{tT}=$ total testosterone $(\mathrm{pg} / \mathrm{ml}), \mathrm{fT}=$ free testosterone $(\mathrm{pg} / \mathrm{ml}), \% \mathrm{fT}=$ relative free testosterone $(\mathrm{fT} / \mathrm{tT}$ as a percentage $)$.

$*$ denotes significance $(p<0.05)$ from respective PRE value; d denotes significance from comparable value in H-EP vs. L-EP; a denotes main effect for phase in all values (H-EP luteal vs. L-EP follicular)

The tT was significantly elevated at POST compared to PRE $(p<0.01)$ following exercise during both phases of the menstrual cycle. At 30POST the responses returned to resting levels and were not different from PRE $(p>0.05)$. Furthermore, the phase of the menstrual cycle did not impact the magnitude of these tT responses to exercise.

Free testosterone (fT) exercise responses, however, were affected by the phases of the menstrual cycle. In the H-EP luteal phase, exercise caused fT to be increased at POST ( $p<$ 0.01 ) compared to PRE, and at 30POST responses trended towards remaining significantly increased from PRE $(p<0.09)$. Conversely, in the L-EP follicular phase no significant changes were observed in fT for the exercise session. Furthermore, the H-EP luteal phase POST fT was significantly different from the L-EP follicular POST (H-EP $>$ L-EP; $p<0.03$ ).

The relative \%fT responses demonstrated a significant effect due to menstrual phase, but not to exercise per se due to variability in the data. That is, a greater portion of $\mathrm{T}$ existed overall in the more biologically active free state (\%fT) during H-EP luteal phase when compared to the L-EP follicular $(p<0.01)$; this finding, however was driven by the increased percentages found at the POST and 30POST in this phase (see Table I). 
The AUC responses are displayed in Table II. The tT AUC responses did not differ between phases of the menstrual cycle. However, for fT during H-EP luteal phase the AUC responses for POST to 30POST as well as TOTAL were significantly greater than during L-EP follicular phase ( $p<0.03$ and $p<0.04$, respectively).

Table II. Area-under-the-curve (AUC) response (mean $\pm \mathrm{SD}$ ) of total and free testosterone to a 60 minute treadmill running sessions in the H-EP and L-EP phases of the menstrual cycle.

\begin{tabular}{|c|c|c|c|c|}
\hline \multicolumn{5}{|c|}{ Hormonal area-under-the-curve responses } \\
\hline \multirow{2}{*}{$\begin{array}{l}\text { Measurement } \\
\text { period }\end{array}$} & \multicolumn{2}{|c|}{ Mid-luteal (H-EP) } & \multicolumn{2}{|c|}{ Mid-follicular (L-EP) } \\
\hline & $\begin{array}{c}\mathrm{tT} \\
(\mathrm{pg} / \mathrm{ml} \times \mathrm{min})\end{array}$ & $\begin{array}{c}\mathbf{f T} \\
(\mathrm{pg} / \mathrm{ml} \times \min )\end{array}$ & $\begin{array}{c}\mathrm{tT} \\
(\mathrm{pg} / \mathrm{ml} \times \min )\end{array}$ & $\begin{array}{c}\text { fT } \\
(\mathrm{pg} / \mathrm{ml} \times \min )\end{array}$ \\
\hline $\begin{array}{l}\text { PRE to POST } \\
\text { AUC }\end{array}$ & $\begin{array}{c}32100 \\
\pm 20475\end{array}$ & $\begin{array}{c}150.0 \\
\pm 127.9\end{array}$ & $\begin{array}{c}28350 \\
\pm 11175\end{array}$ & $\begin{array}{c}91.6 \\
\pm 49.9\end{array}$ \\
\hline $\begin{array}{l}\text { POST to 30POST } \\
\text { AUC }\end{array}$ & $\begin{array}{c}17775 \\
\pm 12150\end{array}$ & $\begin{array}{l}93.5^{\mathrm{d}} \\
\pm 78.1\end{array}$ & $\begin{array}{r}15600 \\
\pm 6825\end{array}$ & $\begin{array}{c}50.8 \\
\pm 30.2\end{array}$ \\
\hline $\begin{array}{l}\text { TOTAL } \\
\text { AUC }\end{array}$ & $\begin{array}{c}50175 \\
\pm 32550\end{array}$ & $\begin{array}{l}243.6^{\mathrm{d}} \\
\pm 204.6\end{array}$ & $\begin{array}{c}43950 \\
\pm 17700\end{array}$ & $\begin{array}{r}142.4 \\
\pm 79.1\end{array}$ \\
\hline
\end{tabular}

$\mathrm{tT}=$ total testosterone $(\mathrm{pg} / \mathrm{ml} \times \mathrm{min}), \mathrm{fT}=$ free testosterone $(\mathrm{pg} / \mathrm{ml} \times \mathrm{min})$;

$\mathrm{d}$ denotes significance from comparable value in H-EP vs. L-EP

\section{Discussion}

The purpose of this study was to examine the $\mathrm{tT}$ and $\mathrm{fT}$ responses to prolonged aerobic exercise during the L-EP and H-EP phases of the menstrual cycle in women. The tT results confirmed and agree with the results of previous studies $(3,8)$. That is, there was no major impact on the tT responses as a result of menstrual phase, only that of exercise. Conversely, the results also indicated that following a prolonged bout of aerobic exercise, the levels of fT are elevated from PRE to POST during the H-EP phase, but without any change during the L-EP phase. Furthermore, the AUC analysis revealed an overall greater fT response in H-EP vs. L-EP. These findings clearly point to the existence of an effect of menstrual cycle phase on the circulating levels of fT in response to exercise, which is a novel finding. Furthermore, the relative \%fT response also exhibited a higher percent of fT overall in the H-EP phase compared to the L-EP phase (N.B., the increased percentages found at POST and 30POST in this phase induced this overall effect).

The precise mechanisms for the elevations of $\mathrm{fT}$ in the absolute (concentrations and AUC) and relative (\%) state in the H-EP phase are currently unclear as the study was not designed to be mechanistic in nature. Nonetheless, a possible explanation could be an increase in glandular $\mathrm{T}$ production, coupled with a potential decrease in hepatic clearance during exercise $(2,5)$. Additionally, greater elevations in core body temperature (CBT) during the H-EP phase (which occurs in eumenorrheic women) may also be a contributing factor (7). Relative to this last point, the elevated CBT in the H-EP phase, may create a decreased affinity of the plasma proteins for testosterone, resulting in greater amounts of biologically active testosterone in the blood (4). Basal body temperature is elevated in the luteal phase (H-EP) of the menstrual cycle and subsequently can lead to great exercise CBT responses $(6,7)$. At this point, further research is necessary in this area to identify the mechanisms behind these findings. 
A limitation to our study was our sample size was small. But, we offset this constraint with a well-executed experimental design as well as for controlling numerous potentially confounding factors, including menstrual cycle timing, time of day, diet, hormonal and exercise training status. These steps strengthened our study and added to the validity of our outcomes.

In conclusion, the present findings point to it being imperative to consider menstrual cycle phase when conducting research involving testosterone in women if researchers are assessing androgenic responses. As previous research has shown, tT exhibits similar increases to a prolonged bout of aerobic exercise regardless of phase $(3,8)$. However, this study shows a more robust increase in the amplitude of fT responses (absolute and relative) during the H-EP luteal phase of the menstrual cycle. This implies the potential for a stronger anabolic action in response to exercise in the luteal phase for eumenorrheic women, which is a topic future research should pursue. Finally, our findings point to the need for researchers, who are examining the $\mathrm{T}$ responses of women, to exercise to examine both $\mathrm{tT}$ and $\mathrm{fT}$ forms in order to have an accurate endocrine assessment.

\section{Acknowledgements}

All authors wish to thank the subjects for their efforts in this study. The authors declare that there are no conflicts of interest that could be perceived as prejudicing the impartiality of the research reported in this manuscript.

\section{REFERENCES}

1. Bunt JC: Metabolic actions of estradiol: significance for acute and chronic exercise responses. Med. Sci. Sports Exerc. 22(3), 286-290 (1990)

2. Cadoux-Hudson TA, Few JD, Imms FJ: The effect of exercise on the production and clearance of testosterone in well-trained young men. Eur. J. Appl. Physiol. 54(3), 321-325 (1985)

3. Enea C, Boisseau N, Ottavy M, Mulliez J, Millet C, Ingrand I, Diaz V, Dugué B: Effects of menstrual cycle, oral contraception, and training on exercise-induced changes in circulating DHEA-sulphate and testosterone in young women. Eur. J. Appl. Physiol. 106(3), 365-373 (2009)

4. Fahrner CL, Hackney AC: Effects of endurance exercise on free testosterone concentration and the binding affinity of sex hormone binding globulin (SHBG). Int. J. Sport Med. 19(1), 12-15 (1998)

5. Hall JE (2009): Neuroendocrine control of the menstrual cycle. In: Yen \& Jaffe's Reproductive Endocrinology: Physiology, Pathophysiology, and Clinical Management, eds Strauss JF, Barbieri RL, $6^{\text {th }}$ ed. Elsevier, New York, pp. 139-154

6. Kolka MA, Stephenson LA: Effect of luteal phase elevation in core temperature on forearm blood flow during exercise. J. Appl. Physiol. 82(4), 1079-1083 (1997)

7. Marsh SA, Jenkins DG: Physiological responses to the menstrual cycle: implications for the development of heat illness in female athletes. Sports Med. 32(10), 601-614 (2002)

8. O’Leary CB, Lehman C, Koltun K, Smith-Ryan A, Hackney AC: Response of testosterone to prolonged aerobic exercise during different phases of the menstrual cycle. Eur. J. Appl. Physiol. 113(9), 2419-2424 (2013)

9. American College of Sports Medicine (2005): ACSM's Guidelines for Exercise Testing and Prescription, $7^{\text {th }}$ ed. Lippincott Williams \& Wilkins, Philadelphia, pp. 287-290 